\title{
Reconstruction of Dielectric Constants of Core and Cladding of Optical Fibers Using Propagation Constants Measurements
}

\author{
E. M. Karchevskii, ${ }^{1}$ A. O. Spiridonov, ${ }^{1}$ A. I. Repina, ${ }^{1}$ and L. Beilina ${ }^{2}$ \\ ${ }^{1}$ Kazan Federal University, Kremlevskaya 18, Kazan 420008, Russia \\ ${ }^{2}$ Chalmers University of Technology and University of Gothenburg, 42196 Gothenburg, Sweden \\ Correspondence should be addressed to E. M. Karchevskii; ekarchev@yandex.ru
}

Received 17 June 2014; Revised 6 September 2014; Accepted 7 September 2014; Published 18 September 2014

Academic Editor: Ernest M. Henley

Copyright (C) 2014 E. M. Karchevskii et al. This is an open access article distributed under the Creative Commons Attribution License, which permits unrestricted use, distribution, and reproduction in any medium, provided the original work is properly cited.

We present new numerical methods for the solution of inverse spectral problem to determine the dielectric constants of core and cladding in optical fibers. These methods use measurements of propagation constants. Our algorithms are based on approximate solution of a nonlinear nonselfadjoint eigenvalue problem for a system of weakly singular integral equations. We study three inverse problems and prove that they are well posed. Our numerical results indicate good accuracy of new algorithms.

\section{Introduction}

In this paper, we investigate the problem of determination of dielectric constants of core and cladding in optical fibers using measurements of propagation constant. Such kinds of problems arise in determination of electromagnetic parameters in nanocomposite materials in nanoengineering. These parameters cannot be measured experimentally because of nanocomposite type of materials [1].

There are many nondestructive material characterization techniques for obtaining permittivities of dielectric materials (see a short review of recent results in [2]). For the first time, the problem of complex permittivity determination in closed rectangular waveguides using propagation constant measurements was investigated in [3]. For open dielectric waveguides of arbitrary cross-section such problems can be set up as inverse eigenvalue problems [4] of the theory of optical waveguides [5]. Problems described in [4] are based on integral formulations of the underlying equations.

Inverse problems for determining of dielectric permittivity were studied in many works; see, for example, [6-9] and references therein. In [10], authors present reconstruction of complex permittivity using finite difference time-domain (FDTD) method. In [11, 12], an analytical method for reconstruction of permittivity of a lossy arbitrary shaped body inside a three-dimensional waveguide using transmitted and reflected data was developed. Results of $[11,12]$ are based on the volume singular integral equation (VSIE) method $[8,9]$ for the system of Maxwell's equations.

The new approximately globally convergent method for reconstruction of permittivity function was developed in [13]. This method was further verified on computationally simulated and on experimental data in [14-22]. An adaptive version of the globally convergent method is developed and computationally verified for the first time in [23]. In recent works $[16,17,21]$, reconstruction of the spatially distributed dielectric functions and shapes of objects placed in the air as well as of buried objects in the dry sand from blind backscattered experimental data using two-stage numerical procedure of $[13,15,24]$ is presented. In the first stage in $[16,21,22]$, the approximately globally convergent method of [13] is applied to get a good first approximation for the exact permittivity function. Then, the local adaptive finite element method of $[25,26]$ is used in the second stage to refine the solution obtained in the first stage. Results of this stage are presented in $[15,17]$. An approximately globally convergent algorithm in a frequency domain for the case of reconstruction of dielectric function in an optical fiber is recently presented in [27].

The method investigated in this work significantly differs from the above described techniques. Compared with [16$21,27]$, we use measurements of the propagation constant 
instead of the electric field data which is typical for waveguides applications. Our new algorithms use solution of integral equation over the boundary of a waveguide and thus they are less computationally expensive compared with methods of $[11,12]$ which are also based on the volume singular integral equation (VSIE) technique [8,9]. The models of [11, 12] require the solution of three-dimensional vector problems and thus they are more computationally demanding and need powerful computer resources.

We note that two mathematical models of optical waveguides were investigated in detail by methods of integral equations in $[19,20,28-34]$. In [19, 20, 28-31], the model of step-index waveguides was studied and, in [32-34], the model of waveguides without a sharp boundary was studied. A review of modern results in this field is given in [35]. To solve our eigenvalue problem, we are not requiring the information about specific values of eigenfunctions. In our algorithms, it is enough only to know that the fundamental mode is excited and then to measure its propagation constant for one or two frequencies. Main applications of our algorithm are, for example, in detection of defects in metamaterials and in nanoelectronics [36,37], calculation of dielectric constant of saline water $[38,39]$, and in microwave imaging technology in remote sensing [40].

An outline of this paper is a follows. In Section 2, we first present a mathematical model of a weakly guiding stepindex arbitrarily shaped optical fiber and formulate three inverse spectral problems. Then, we present new numerical algorithms for calculation of dielectric constants based on an approximate solution of a nonlinear nonselfadjoint eigenvalue problem for a system of weakly singular integral equations. In Section 3, we present reconstruction results which show efficiency and robustness of new developed algorithms.

\section{Methods of Solution}

2.1. Nonlinear Eigenvalue Problem for Transverse Wavenumbers. Let us consider an optical fiber as a regular cylindrical dielectric waveguide in a free space. The cross-section of the waveguide's core is a bounded domain $\Omega_{i}$ with twice continuously differentiable boundary $\gamma$ (see Figure 1). The axis of the cylinder is parallel to the $x_{3}$-axis. Let $\Omega_{e}=$ $R^{2} \backslash \bar{\Omega}_{i}$ be the unbounded domain of the cladding. Let the permittivity be prescribed as a positive piecewise constant function $\varepsilon$ which is equal to a constant $\varepsilon_{\infty}$ in the domain $\Omega_{e}$ and to a constant $\varepsilon_{+}>\varepsilon_{\infty}$ in the domain $\Omega_{i}$.

Eigenvalue problems of optical waveguide theory [5] are formulated on the base of the set of homogeneous Maxwell equations:

$$
\operatorname{rot} \mathscr{E}=-\mu_{0} \frac{\partial \mathscr{H}}{\partial t}, \quad \operatorname{rot} \mathscr{H}=\varepsilon_{0} \varepsilon \frac{\partial \mathscr{E}}{\partial t} .
$$

Here, $\mathscr{E}$ and $\mathscr{H}$ are electric and magnetic field vectors and $\varepsilon_{0}$ and $\mu_{0}$ are the free-space dielectric and magnetic constants. Nontrivial solutions of set (1), which have the form

$$
\left[\begin{array}{l}
\mathscr{E} \\
\mathscr{H}
\end{array}\right]\left(x, x_{3}, t\right)=\operatorname{Re}\left(\left[\begin{array}{l}
\mathrm{E} \\
\mathrm{H}
\end{array}\right](x) e^{i\left(\beta x_{3}-\omega t\right)}\right),
$$

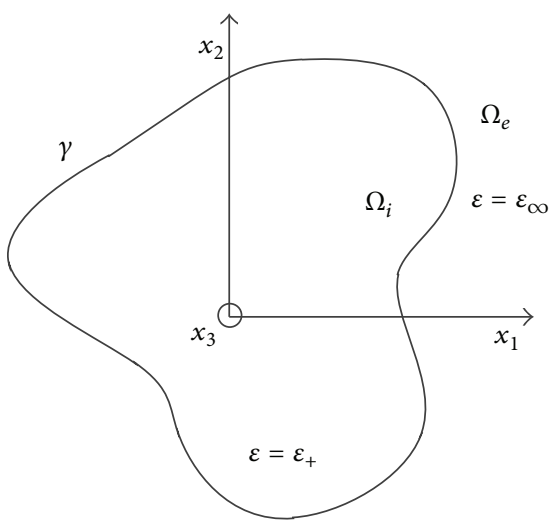

FIGURE 1: The cross-section of a cylindrical dielectric waveguide in a free space.

are called the eigenmodes of the waveguide. Here, positive $\omega$ is the radian frequency, $\beta$ is the propagation constant, $\mathrm{E}$ and $\mathrm{H}$ are complex amplitudes of $\mathscr{E}$ and $\mathscr{H}$, and $x=\left(x_{1}, x_{2}\right)$.

In forward eigenvalue problems, the permittivity is known and it is necessary to calculate longitudinal wavenumbers $k=\omega \sqrt{\varepsilon_{0} \mu_{0}}$ and propagation constants $\beta$ such that there exist eigenmodes. The eigenmodes have to satisfy to a transparency condition at the boundary $\gamma$ and to a condition at infinity.

In inverse eigenvalue problems, it is necessary to reconstruct the unknown permittivity $\varepsilon$ by some information on natural eigenmodes which exist for some eigenvalues $k$ and $\beta$. The main question is how many observations of natural eigenmodes are enough for unique and stable reconstruction of the permittivity.

The domain $\Omega_{e}$ is unbounded. Therefore, it is necessary to formulate a condition at infinity for complex amplitudes $\mathrm{E}$ and $\mathrm{H}$ of eigenmodes. Let us confine ourselves to the investigation of the surface modes only. The propagation constants $\beta$ of surface modes are real and belong to the interval $G=\left(k \varepsilon_{\infty}, k \varepsilon_{+}\right)$. The amplitudes of surface modes satisfy the following condition:

$$
\left[\begin{array}{l}
\mathrm{E} \\
\mathrm{H}
\end{array}\right]=e^{-\sigma r} \mathrm{O}\left(\frac{1}{\sqrt{r}}\right), \quad r=|x| \longrightarrow \infty .
$$

Here, $\sigma=\sqrt{\beta^{2}-k^{2} \varepsilon_{\infty}}>0$ is the transverse wavenumber in the cladding.

Denote by $\chi=\sqrt{k^{2} \varepsilon_{+}-\beta^{2}}$ the transverse wavenumber in the waveguide's core. Under the weakly guidance approximation [5], the original problem was reduced in [29] to the calculation of numbers $\chi$ and $\sigma$ such that there exist nontrivial solutions $u=H_{1}=H_{2}$ of Helmholtz equations:

$$
\begin{aligned}
& \Delta u+\chi^{2} u=0, \quad x \in \Omega_{i}, \\
& \Delta u-\sigma^{2} u=0, \quad x \in \Omega_{e},
\end{aligned}
$$

which satisfy the transparency conditions

$$
u^{+}=u^{-}, \quad \frac{\partial u^{+}}{\partial v}=\frac{\partial u^{-}}{\partial v}, \quad x \in \gamma .
$$


Here, $v$ is the normal derivative on $\gamma, u^{-}$(resp., $u^{+}$) is the limit value of a function $u$ from the interior (resp., the exterior) of $\gamma$.

Denote by $U$ the space of continuous and continuously differentiable functions in $\Omega_{i}$ and $\Omega_{e}$ and twice continuously differentiable functions in $\Omega_{i}$ and $\Omega_{e}$, satisfying to condition (3). Let us calculate nontrivial solutions $u$ of problem (4)-(5) in the space $U$.

Let $\sigma>0$ be a given number. A nonzero function $u \in U$ is called an eigenfunction of problem (4)-(5) corresponding to a real eigenvalue $\chi$ if relationships (4)-(5) hold.

The next theorem follows from results of [29].

Theorem 1. For any $\sigma>0$, the eigenvalues $\chi$ of problem (4)-(5) can be only positive isolated numbers. Each number $\chi$ depends continuously on $\sigma$.

For waveguides of circular cross-section, the analogous results about the localization of the surface modes spectrum and about the continuous dependence between the transverse wavenumbers $\sigma$ and $\chi$ were obtained in [5]. The results of [5] are valid only for waveguides of circular cross-section by the method of separation of variables. Theorem 1 generalizes the results of [5] for the case of an arbitrary smooth boundary. The next theorem follows from results of [34] (see an illustration in Figure 2).

Theorem 2. The following statements hold.

(1) For any $\sigma>0$, there exist the denumerable set of positive eigenvalues $\chi_{i}(\sigma)$, where $i=1,2, \ldots$, of a finite multiplicity with only cumulative point at infinity.

(2) For any $\sigma>0$, the smallest eigenvalue $\chi_{1}(\sigma)$ is positive and simple (its multiplicity is equal to one); corresponding eigenfunction $u_{1}$ can be chosen as the positive function in the domain $\Omega_{i}$.

(3) $\chi_{1}(\sigma) \rightarrow 0$ at $\sigma \rightarrow 0$.

For a given $\sigma>0$, the smallest eigenvalue $\chi_{1}(\sigma)$ and corresponding eigenfunction $u_{1}$ define the eigenmode which is called the fundamental mode (see the bottom curve plotted by the red solid line in Figure 2). Thus, Theorem 2 states, particularly, that, for any $\sigma>0$, there exists exactly one fundamental mode.

To compute eigenmodes, we use the representation of eigenfunctions of problem (4)-(5) in the form of single-layer potentials [41]:

$$
\begin{aligned}
& u(x)=\int_{\gamma} \Phi(\chi ; x, y) f(y) d l(y), \quad x \in \Omega_{i}, \\
& u(x)=\int_{\gamma} \Psi(\sigma ; x, y) g(y) d l(y), \quad x \in \Omega_{e} .
\end{aligned}
$$

Here,

$$
\begin{aligned}
& \Phi(\chi ; x, y)=\frac{i}{4} H_{0}^{(1)}(\chi|x-y|), \\
& \Psi(\sigma ; x, y)=\frac{1}{2 \pi} K_{0}(\sigma|x-y|)
\end{aligned}
$$

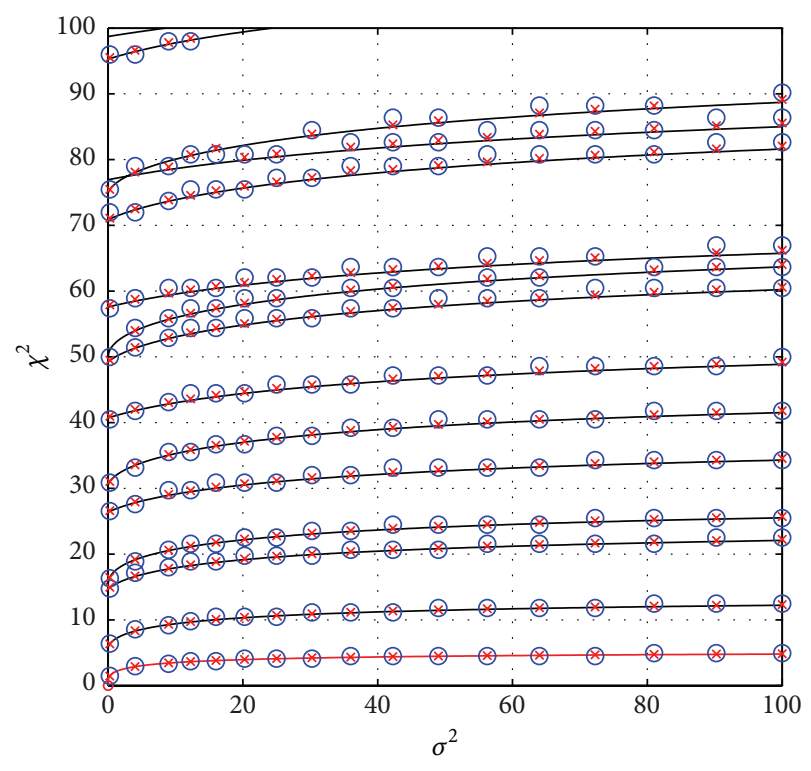

Figure 2: The plot of the dispersion curves computed by the spline-collocation method for surface eigenmodes in a weakly guiding dielectric waveguide of the circular cross-section. The exact solutions are plotted by solid lines. The solution for the fundamental mode is plotted by the lower red solid line. Numerical solutions of the residual inverse iteration method are marked by red crosses. Initial approximations for SVDs are marked by blue circles.

are the fundamental solutions of Helmholtz equations (4) correspondingly, $H_{0}^{(1)}(z)$ is Hankel function of the first kind, $K_{0}(z)$ is Macdonald function, and unknown densities $f$ and $g$ belong to the Hölder space $C^{0, \alpha}(\gamma)$. Then, $f$ and $g$ satisfy to the following system of boundary integral equations for $x \in \gamma$ :

$$
\begin{aligned}
& \mathscr{A}_{11}(\chi) f+\mathscr{A}_{12}(\sigma) g=0, \\
& \mathscr{A}_{21}(\chi) f+\mathscr{A}_{22}(\sigma) g=0,
\end{aligned}
$$

where

$$
\begin{aligned}
& \left(\mathscr{A}_{11}(\chi) f\right)(x)=\int_{\gamma} \Phi(\chi ; x, y) f(y) d l(y), \\
& \left(\mathscr{A}_{12}(\sigma) g\right)(x)=-\int_{\gamma} \Psi(\sigma ; x, y) g(y) d l(y), \\
& \left(\mathscr{A}_{21}(\chi) f\right)(x)=\frac{1}{2} f(x)+\int_{\gamma} \frac{\partial \Phi(\chi ; x, y)}{\partial \nu(x)} f(y) d l(y), \\
& \left(\mathscr{A}_{22}(\sigma) g\right)(x)=\frac{1}{2} g(x)-\int_{\gamma} \frac{\partial \Psi(\sigma ; x, y)}{\partial \nu(x)} g(y) d l(y) .
\end{aligned}
$$

Suppose that the boundary $\gamma$ is parametrically defined by a function $r=r(t)$ of $t \in[0,2 \pi]$ and this parametrization is regular. We consider functions from $C^{0, \alpha}(\gamma)$ and $C^{1, \alpha}(\gamma)$ as Hölder-continues and Hölder-continuously differentiable $2 \pi$-periodic functions of parameter $t$. Then, for $x=x(t) \in \gamma$, 
where $t \in[0,2 \pi]$, system (8) is transformed to the following system of equations:

$$
\begin{aligned}
& L p_{1}+B_{11}(\chi, \sigma) p_{1}+B_{12}(\chi, \sigma) p_{2}=0 \\
& p_{2}+B_{21}(\chi, \sigma) p_{1}+B_{22}(\chi, \sigma) p_{2}=0
\end{aligned}
$$

Here, new unknown functions are defined as follows:

$$
\begin{array}{r}
p_{1}(\tau)=(f(y)-g(y))\left|r^{\prime}(\tau)\right|, \quad p_{2}(\tau)=f(y)+g(y), \\
y=y(\tau), \quad \tau \in[0,2 \pi] .
\end{array}
$$

Integral operators are defined by the following relationships for $t \in[0,2 \pi]$ :

$$
\begin{gathered}
\left(L p_{1}\right)(t)=-\frac{1}{2 \pi} \int_{0}^{2 \pi} \ln \left|\sin \frac{t-\tau}{2}\right| p_{1}(\tau) d \tau, \\
\left(B_{i j}(\chi, \sigma) p^{(j)}\right)(t)=\frac{1}{2 \pi} \int_{0}^{2 \pi} h_{i j}(\chi, \sigma ; t, \tau) p^{(j)}(\tau) d \tau,
\end{gathered}
$$

where

$$
\begin{aligned}
& h_{11}(\chi, \sigma ; t, \tau)=2 \pi\left(G_{11}(\chi ; t, \tau)+G_{12}(\sigma ; t, \tau)\right), \\
& h_{12}(\chi, \sigma ; t, \tau)=2 \pi\left(G_{11}(\chi ; t, \tau)-G_{12}(\sigma ; t, \tau)\right)\left|r^{\prime}(\tau)\right|, \\
& h_{21}(\chi, \sigma ; t, \tau)=4 \pi\left(G_{21}(\chi ; t, \tau)+G_{22}(\sigma ; t, \tau)\right), \\
& h_{22}(\chi, \sigma ; t, \tau)=4 \pi\left(G_{21}(\chi ; t, \tau)-G_{22}(\sigma ; t, \tau)\right)\left|r^{\prime}(\tau)\right|, \\
& G_{11}(\chi ; t, \tau)=\Phi(\chi ; x, y)+\frac{1}{2 \pi} \ln \left|\sin \frac{t-\tau}{2}\right|, \\
& G_{12}(\sigma ; t, \tau)=\Psi(\sigma ; x, y)+\frac{1}{2 \pi} \ln \left|\sin \frac{t-\tau}{2}\right|, \\
& G_{21}(\chi ; t, \tau)=\frac{\partial \Phi(\chi ; x, y)}{\partial \nu(x)}, \\
& G_{22}(\sigma ; t, \tau)=\frac{\partial \Psi(\sigma ; x, y)}{\partial \nu(x)} .
\end{aligned}
$$

The linear operator $L: C^{0, \alpha}(\gamma) \rightarrow C^{1, \alpha}(\gamma)$ is continuous and continuously invertible (for details, see, e.g., [29]). It was proved in [29] that, for each $\chi>0, \sigma>0$, the linear operators

$$
\begin{array}{ll}
B_{21}(\chi, \sigma), & B_{22}(\chi, \sigma): C^{0, \alpha}(\gamma) \longrightarrow C^{0, \alpha}(\gamma), \\
B_{11}(\chi, \sigma), & B_{12}(\chi, \sigma): C^{0, \alpha}(\gamma) \longrightarrow C^{1, \alpha}(\gamma)
\end{array}
$$

are compact. Therefore, system (8) is equivalent to the following Fredholm operator equation of the second kind:

$$
A(\chi, \sigma) w=(I+B(\chi, \sigma)) w=0,
$$

where $w=\left(w_{1}, w_{2}\right)^{T}$,

$$
w_{1}=L p_{1} \in C^{1, \alpha}(\gamma), \quad w_{2}=p_{2} \in C^{0, \alpha}(\gamma),
$$

and the compact operator $B$ acting in the Banach space $W=$ $C^{1, \alpha}(\gamma) \times C^{0, \alpha}(\gamma)$ is defined as follows:

$$
B w=\left[\begin{array}{ll}
B_{11} L^{-1} & B_{12} \\
B_{21} L^{-1} & B_{22}
\end{array}\right]\left[\begin{array}{l}
w_{1} \\
w_{2}
\end{array}\right] ;
$$

by $I$ the identical operator is denoted.

Let $\sigma>0$ be a given number. A nonzero element $w \in W$ is called an eigenfunction of the operator-valued function $A(\chi)$ corresponding to a characteristic value $\chi>0$, if (15) holds.

It follows from results of [29] that original problem (4)-(5) is spectrally equivalent to (15). Namely, the following theorem is true.

Theorem 3. Let $\sigma>0$ be a given number. The following statements hold.

(1) If a function $w$ is the eigenfunction of the operatorvalued function $A(\chi)$ corresponding to a characteristic value $\chi_{0}>0$, then the function $u$ defined by equalities (6), where $\chi=\chi_{0}$,

$$
\begin{aligned}
& f=\frac{w_{2}}{2}+\frac{L^{-1} w_{1}}{2\left|r^{\prime}\right|}, \\
& g=\frac{w_{2}}{2}-\frac{L^{-1} w_{1}}{2\left|r^{\prime}\right|},
\end{aligned}
$$

is the eigenfunction of problem (4)-(5) corresponding to the eigenvalue $\chi_{0}$.

(2) Each eigenfunction of problem (4)-(5) corresponding to an eigenvalue $\chi_{0}>0$ can be represented in the form of singlelayer potentials (6) with some Hölder-continuous densities $f$ and $g$, respectively. At the same time, the function

$$
w=\left(L\left((f-g)\left|r^{\prime}\right|\right), f+g\right)
$$

is the eigenfunction of the operator-valued function $A(\chi)$ corresponding to the characteristic value $\chi_{0}$.

Let us formulate the nonlinear spectral problem for transverse wavenumbers as follows. Suppose that the boundary $\gamma$ of the waveguide's cross-section and the number $\sigma>0$ are given. It is necessary to calculate all characteristic values $\chi$ of the operator-valued function $A(\chi)$ in some given interval.

A spline-collocation method was proposed in [31] for numerical calculations of characteristic values $\chi$ of the operator-valued function $A(\chi)$ for given $\sigma$, and problem (15) for each fixed $\sigma$ was reduced to a nonlinear finite-dimensional eigenvalue problem of the form

$$
A_{n}(\chi) w_{n}=0
$$

where $n$ is the number of collocation points. For numerical solution of obtained nonlinear finite-dimensional eigenvalue problem, we use the residual inverse iteration method [42].

Any iterative numerical method for computations of the nonlinear eigenvalues $\chi$ needs good initial approximations for each given $\sigma$. Usually initial approximations are chosen by a physical intuition using prior information on solutions. If we model fundamentally new types of waveguides, solve 
inverse problems, or investigate defects in fibers, then we may not have accurate prior information on solutions.

In this case, we can investigate spectral properties of the matrix $A_{n}(\chi)$ as a function of variable $\chi$ for each fixed $\sigma$. For each given point in an investigated domain of parameters $\chi$ and $\sigma$, we calculate the condition number of matrix $A_{n}$ :

$$
\text { cond }\left(A_{n}(\chi)\right)=\frac{\rho_{1}}{\rho_{n}}
$$

where $\rho_{1}$ and $\rho_{n}$ are maximal and minimal singular values of matrix. If for given $\sigma$ a number $\chi$ is equal to a nonlinear eigenvalue of $A_{n}(\chi)$, then the condition number is equal to infinity. Therefore, the numbers $\chi$ for which the condition number is big enough are good approximations for eigenvalues. Singular values are calculated by singular value decomposition (SVD) method:

$$
A_{n}(\chi)=U S V, \quad S=\operatorname{diag}\left(\rho_{1}, \rho_{2}, \ldots, \rho_{n}\right),
$$

where $U, V$ are unitary matrices and $S$ is a diagonal matrix; the singular numbers form $S$. The calculations are based on unitary transformations of the matrix $A$ and therefore are stable.

In the next step, for each $\sigma$ in the investigated interval, we use obtained initial approximations for numerical calculations of nonlinear eigenvalues $\chi$ by the residual inverse iteration method.

On the base of solution of nonlinear eigenvalue problem (15) for transverse wavenumbers, we solve the forward and inverse spectral problems for weakly guiding step-index waveguides.

2.2. Forward Spectral Problem. Clearly, if for given $\sigma$ the characteristic values $\chi$ of the operator-valued function $A(\chi)$ were calculated and also if the permittivities $\varepsilon_{+}, \varepsilon_{\infty}$ are known, then the longitudinal wavenumber $k$ and the propagation constant $\beta$ are calculated by the following explicit formulas:

$$
k^{2}=\frac{\sigma^{2}+\chi^{2}}{\varepsilon_{+}-\varepsilon_{\infty}}, \quad \beta^{2}=\frac{\varepsilon_{+} \sigma^{2}+\varepsilon_{\infty} \chi^{2}}{\varepsilon_{+}-\varepsilon_{\infty}} .
$$

For each given $\varepsilon_{+}, \varepsilon_{\infty}$, and $i$, the function $\chi_{i}(\sigma)$ generates a function $\beta^{2}$ of variable $k^{2}$. As an example in Figure 3, we present the plot of the computed function $\beta^{2}=\beta^{2}\left(k^{2}\right)$ corresponding to the fundamental mode (see the bottom curve plotted by the red solid line in Figure 2) of the circular waveguide. Here, $\varepsilon_{+}=2, \varepsilon_{\infty}=1$, and $i=1$. We will use two points marked by circle and by square in the next sections as test points for numerical solution of inverse spectral problems.

2.3. Inverse Spectral Problems. In this subsection, we present three algorithms for approximate solution of three inverse spectral problems. The algorithms are based on previous numerical solution of nonlinear eigenvalue problem (15) for transverse wavenumbers, namely, on numerical calculations of characteristic values $\chi_{1}(\sigma)$ of the operator-valued function $A(\chi)$ for fundamental mode and for $\sigma$ in an appropriate interval.

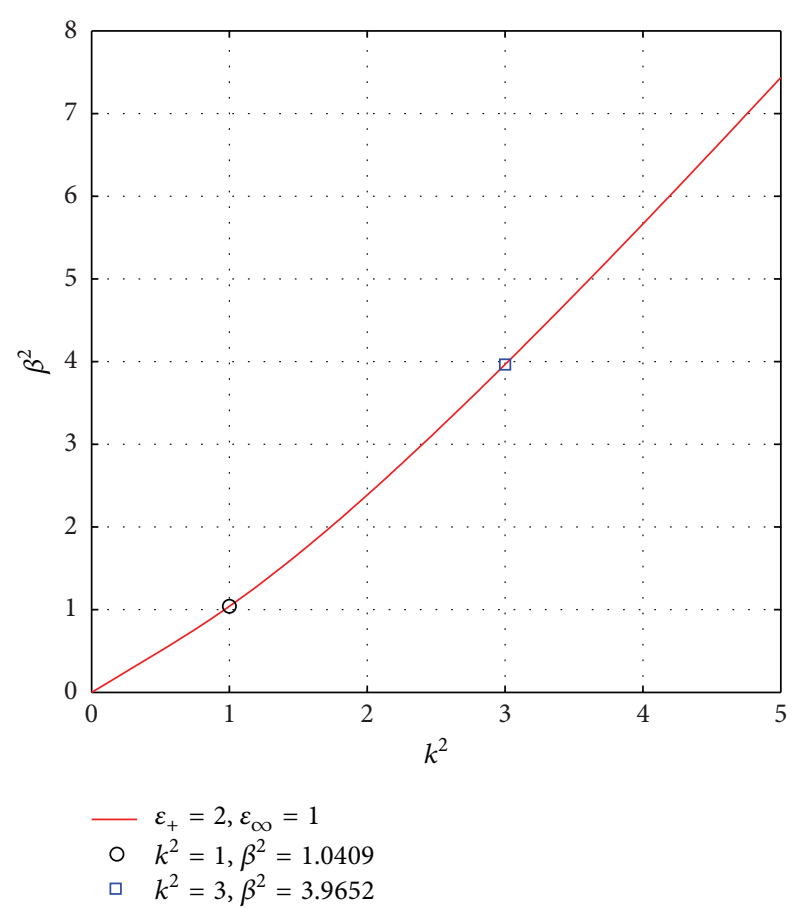

Figure 3: The red solid line presents the plot of the computed function $\beta^{2}=\beta^{2}\left(k^{2}\right)$ corresponding to the fundamental mode of the circular waveguide.

2.3.1. Algorithm for Reconstruction of the Permittivity of the Core. The first inverse problem is formulated as follows. Suppose that the boundary $\gamma$ of the waveguide's cross-section and the permittivity $\varepsilon_{\infty}$ of the cladding are given. Suppose that the propagation constant $\beta$ of the fundamental mode is measured for a given wavenumber $k$. The measuring can be done by experimental methods described, for example, in [3]. It is necessary to calculate the permittivity $\varepsilon_{+}$of the waveguide's core.

The solution of this inverse spectral problem is calculated in the following way. First, we compute the number

$$
\sigma=\sqrt{\beta^{2}-k^{2} \varepsilon_{\infty}}
$$

which is calculated for given values of $\beta, k$, and $\varepsilon_{\infty}$. Then, the transverse wavenumber $\chi(\sigma)$ is calculated by the splinecollocation method for the obtained $\sigma$ of the fundamental mode. This number is calculated by an interpolation of the function $\chi_{1}(\sigma)$ with respect to the points obtained when the nonlinear spectral problem for transverse wavenumbers was numerically solved. Finally, the permittivity of the waveguide's core is calculated by the following explicit formula:

$$
\varepsilon_{+}=\frac{\chi^{2}+\beta^{2}}{k^{2}} .
$$

2.3.2. Algorithm for Reconstruction of the Permittivity of the Cladding. Suppose that the permittivity of the core is given and that the propagation constant $\beta$ of the fundamental mode is measured for a given wavenumber $k$. It is necessary to calculate the permittivity $\varepsilon_{\infty}$ of the waveguide's cladding. 


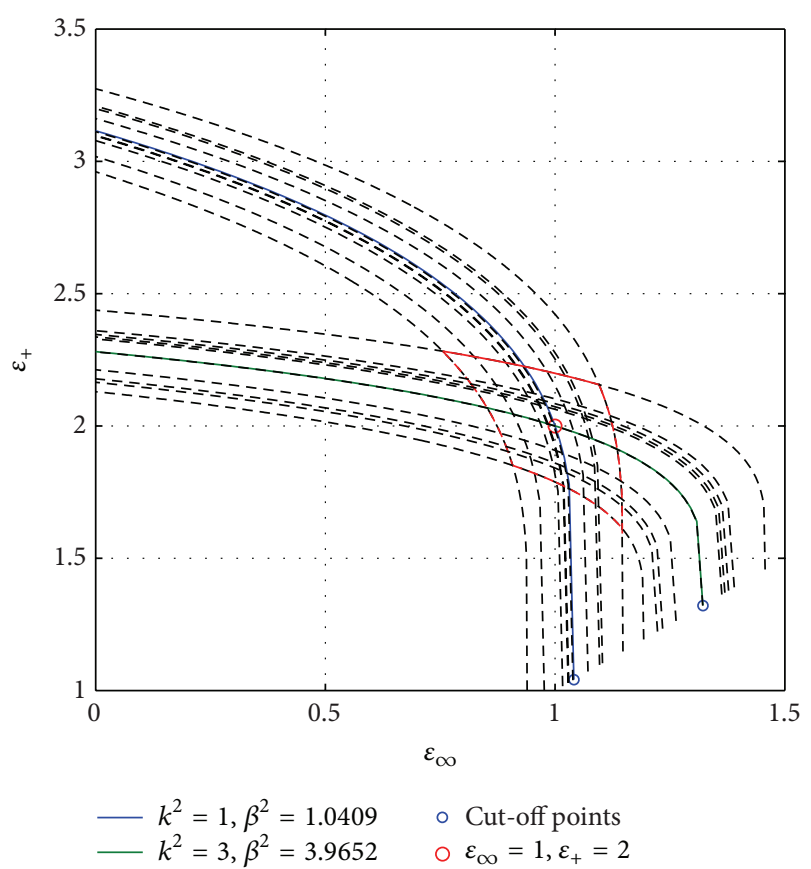

FIgURE 4: The blue and the green solid lines are plots of function $\varepsilon_{+}=\varepsilon_{+}\left(\varepsilon_{\infty}\right)$ for given pairs of $k$ and $\beta$ of the fundamental mode. The unique exact solution of the inverse spectral problem is marked by the red circle. The approximate solution obtained by the splinecollocation method coincides with the red circle for the exact $\beta$. For randomly noised $\widetilde{\beta}$, approximate solutions are intersections of dashed lines. They belong to the red rhomb.

The solution of this problem is calculated in the following way. First, we compute the number

$$
\chi=\sqrt{k^{2} \varepsilon_{+}-\beta^{2}}
$$

which is calculated for given values of $\beta, k$, and $\varepsilon_{+}$. Second, the transverse wavenumber $\sigma$ is calculated by the splinecollocation method for the obtained $\chi$ for the fundamental mode. Third, the permittivity of the waveguide's cladding is calculated by the following explicit formula:

$$
\varepsilon_{\infty}=\frac{\beta^{2}-\sigma^{2}}{k^{2}} .
$$

\subsubsection{Algorithm for Reconstruction of Two Permittivities of the} Core and of the Cladding. The full variant of our problem is the reconstruction of both permittivities of the core and of the cladding. The solution for the fundamental mode of nonlinear eigenvalue problem (15) for transverse wavenumbers gives us an implicit function $\varepsilon_{+}$of the variable $\varepsilon_{\infty}$ for each fixed longitudinal wavenumber $k$ and propagation constant $\beta$. For example, in Figure 4, the blue and the green solid lines are plots of this function for given pairs of $k$ and $\beta$.

The intersection of these lines marked by the red circle is the unique exact solution of our problem. Therefore, we calculate the permittivities $\varepsilon_{+}$and $\varepsilon_{\infty}$ as the solution of the following nonlinear system of two equations:

$$
\begin{aligned}
& \chi\left(\sqrt{\beta_{1}^{2}-k_{1}^{2} \varepsilon_{\infty}}\right)=\sqrt{k_{1}^{2} \varepsilon_{+}-\beta_{1}^{2}}, \\
& \chi\left(\sqrt{\beta_{2}^{2}-k_{2}^{2} \varepsilon_{\infty}}\right)=\sqrt{k_{2}^{2} \varepsilon_{+}-\beta_{2}^{2}} .
\end{aligned}
$$

Here, $k_{1}$ and $k_{2}$ are some given distinct longitudinal wavenumbers, $\beta_{1}$ and $\beta_{2}$ are corresponding measured propagation constants, and $\chi$ is the function of variable $\varepsilon_{\infty}$ for fixed $k_{j}$ and $\beta_{j}, j=1,2$.

\section{Results and Discussion}

Let us describe numerical results obtained for a nonlinear eigenvalue problem (15) for transverse wavenumbers. We compare our numerical results with the well-known exact solution for the circular waveguide obtained by the method of separation of variables (see, e.g., [5]). In Figure 2, we present some dispersion curves for surface eigenwaves of the circular waveguide. Here, the exact solution is plotted by solid lines.

We started our numerical calculations with computations of initial approximations for nonlinear eigenvalues $\chi$. To do that, we have used SVD as was described in Section 2.1. Calculated initial approximations are marked in Figure 2 by blue circles. The blue circles are only the initial approximations for eigenvalues $\chi$. We apply these initial approximations as start points in the residual inverse iteration method. Using this iteration method, for each given $\sigma$, we solved numerically the finite-dimensional nonlinear eigenvalue problem (20) depending on eigenvalues $\chi$. The numerical solution which resulted in this method is marked in Figure 2 by red crosses.

Note here that, applying previously calculated initial approximations for nonlinear eigenvalues $\chi$, we numerically solve this problem directly and without any prior physical information. Figure 2 illustrates that the initial approximations are good enough and we can use them in the majority of cases without iterative refinement of solutions.

Our next numerical experiment shows reconstruction of core's permittivity using the algorithm in Section 2.3.1. The mathematical analysis of existence of the solution of the original spectral problem (4)-(5) for transverse wavenumbers is presented in Theorems 1 and 2. An illustration of the theoretical results is shown in Figure 2. Analyzing Figure 2, we observe that the fundamental mode (see the red solid curve in Figure 2) exists for each wavenumber $k>0$. The fundamental mode is unique; its dispersion curve does not intersect with any other curves and well separated from them. Therefore, the inverse spectral problem's solution exists and is unique for each wavenumber $k>0$; this solution depends continuously on given data. In other words, the inverse spectral problem is well posed by Hadamard. We can see an example of this continuity dependence in Figure 5. The red solid line is the plot of the function $\varepsilon_{+}$of $\beta^{2}$ for the fundamental mode. 


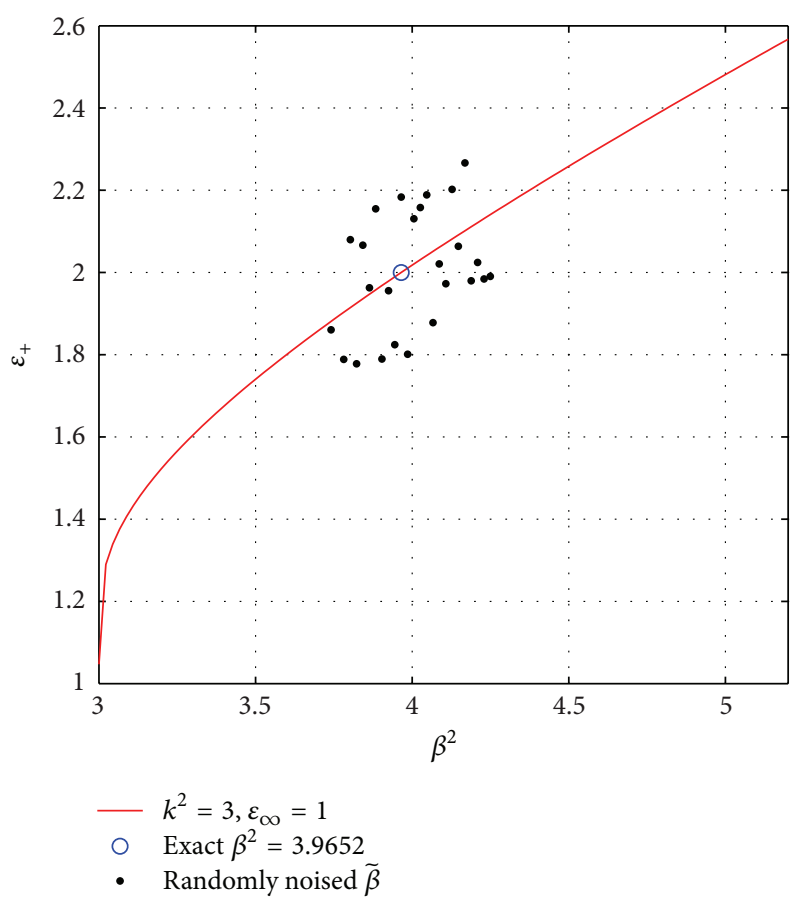

Figure 5: The red solid line is the plot of function $\varepsilon_{+}=\varepsilon_{+}\left(\beta^{2}\right)$ for the fundamental mode. The approximate solution obtained by the spline-collocation method is marked by the blue circle for the exact $\beta$ and by black points for the randomly noised $\widetilde{\beta}$.

In our computations, by analogy with $[13,14]$, we have introduced random noise in the propagation constant as

$$
\widetilde{\beta}=\beta(1+p \alpha)
$$

where $\beta=3.98518$ is the exact measured propagation constant, $\alpha \in(-1,1)$ are randomly distributed numbers, and $p$ is the noise level. In our computations, we have used $p=0.05$ and, thus, the noise level was $5 \%$.

Some numerical results of reconstruction of $\varepsilon_{+}$are presented in Figure 5. The approximated value of $\varepsilon_{+}$for the noise-free data is marked in Figure 5 by the blue circle. Approximated values of $\varepsilon_{+}$for randomly distributed noise $\widetilde{\beta}$ with the $5 \%$ noise level are marked in Figure 5 by the black points. Using this figure, we observe that the approximate solutions even for the randomly noised $\widetilde{\beta}$ were stable. We can conclude that, for the unique and stable reconstruction of the constant waveguide permittivity $\varepsilon_{+}$, it is enough to measure the propagation constant $\beta$ of the fundamental mode for only one wavenumber $k$.

Absolutely analogous results were obtained for reconstruction of cladding's permittivity using the algorithm in Section 2.3.2. As in Figure 5, the red solid line in Figure 6 is the plot of continuous function $\varepsilon_{\infty}$ of squared $\beta$ for the fundamental mode. The approximate solution obtained by the spline-collocation method is marked by the blue circle for the exact $\beta$ and by black points for the randomly noised $\tilde{\beta}$. Using this figure, we observe that the approximate solutions for the randomly noised $\widetilde{\beta}$ were stable in this case too. For

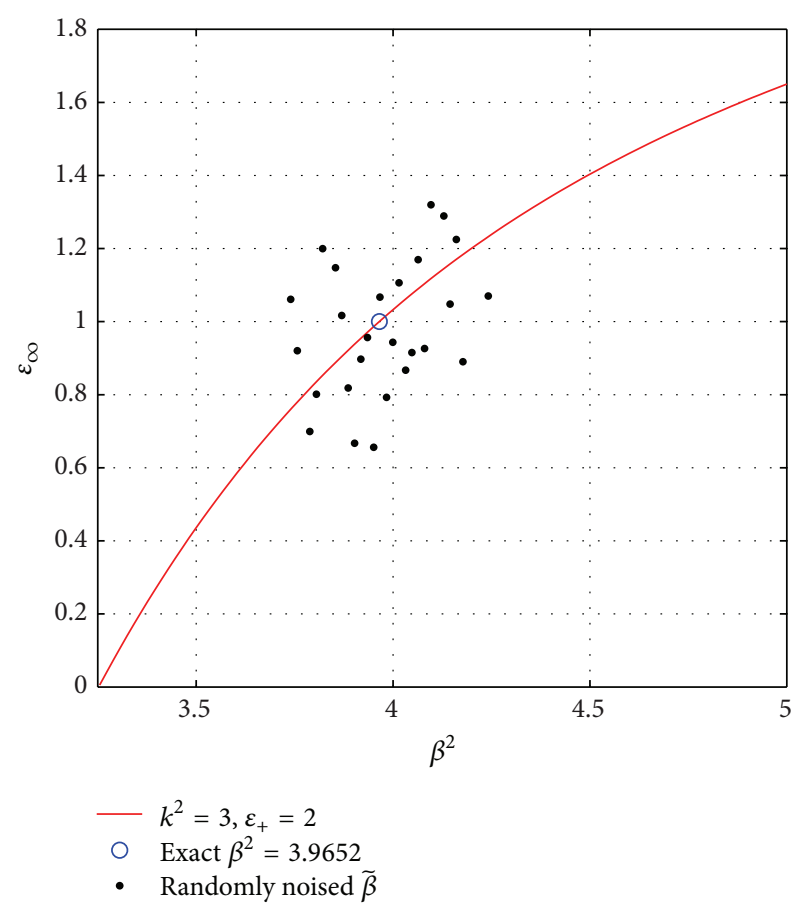

FIGURE 6: The red solid line is the plot of function $\varepsilon_{\infty}=\varepsilon_{\infty}\left(\beta^{2}\right)$ for the fundamental mode. The approximate solution obtained by the spline-collocation method is marked by the blue circle for the exact $\beta$ and by black points for the randomly noised $\widetilde{\beta}$.

this test, we can also conclude that, for the unique and stable reconstruction of the constant permittivity $\varepsilon_{\infty}$, it is enough to measure the propagation constant $\beta$ of the fundamental mode for only one wavenumber $k$.

Finally, we show simultaneous reconstruction of both permittivities of core and of cladding using the algorithm in Section 2.3.3. The approximate solution obtained by the spline-collocation method is marked in Figure 4 by the red circle for the exact propagation constant. We have introduced the random noise in the propagation constant similarly with above described tests. The approximate solutions for the noised $\widetilde{\beta}$ are presented in Figure 4 as intersections of dashed lines. Using Figure 4, we observe that the approximate solutions for the randomly noised $\beta$ belong to the red rhomb and are stable. Therefore, we can conclude that, for the unique and stable reconstruction of the dielectric constants $\varepsilon_{\infty}$ and $\varepsilon_{+}$, it is enough to measure the propagation constant $\beta$ of the fundamental mode for two distinct wavenumbers $k$.

\section{Conclusion}

In this work, we have formulated three inverse spectral problems and proved that they are well posed. It is important to note that, in our algorithms, any information on specific values of eigenfunctions is not required. For solution of these inverse problems, it is enough to know that the fundamental mode is excited and then to measure its propagation constant for one or for two frequencies. This approach satisfies 
the practice of physical experiments because usually the fundamental mode is excited in waveguides in real applications [3]. Moreover, the fundamental mode can be excited only for enough wide range of frequencies.

For the approximate solution of the inverse problems, we propose to solve the nonlinear spectral problem for transverse wavenumbers in order to compute the dispersion curve for the fundamental mode. These calculations are done accurately by the spline-collocation method. Finally, we have demonstrated unique and stable reconstruction of the permittivities using new inverse algorithms.

\section{Conflict of Interests}

The authors declare that there is no conflict of interests regarding the publication of this paper.

\section{Acknowledgments}

The authors would like to thank Michael Havrilla and George Hanson for helpful discussions concerning the topics covered in this work. This work was funded by the subsidy allocated to Kazan Federal University for the state assignment in the sphere of scientific activities; the work was supported also by RFBR and by Government of Republic Tatarstan, Grant 12-01-97012-r_povolzh'e_a. The research of Larisa Beilina was supported by the Swedish Research Council, the Swedish Foundation for Strategic Research (SSF) through the Gothenburg Mathematical Modelling Centre (GMMC), and by the Swedish Institute.

\section{References}

[1] L. Solymar and E. Shamonina, Waves in Metamaterials, Oxford University Press, Oxford, UK.

[2] M. Havrilla, A. Bogle, M. Hyde IV, and E. Rothwell, "EM material characterization of conductor backed media using a NDE microstrip probe," Studies in Applied Electromagnetics and Mechanics, vol. 38, no. 2, pp. 210-218, 2014.

[3] M. D. Janezic and J. A. Jargon, "Complex permittivity determination from propagation constant measurements," IEEE Microwave and Wireless Components Letters, vol. 9, no. 2, pp. 76-78, 1999.

[4] M. T. Chu and G. H. Golub, Inverse Eigenvalue Problems: Theory, Algorithms, and Applications, Oxford University Press, 2005.

[5] A. W. Snyder and J. D. Love, Optical Waveguide Theory, Chapman \& Hall, London, UK, 1983.

[6] G. Nakamura and M. Sini, "On the near field measurement for the inverse scattering problem for ocean acoustics," Inverse Problems, vol. 20, no. 5, pp. 1387-1392, 2004.

[7] A. Ramm, Multidimensional Inverse Scattering Problems, Wiley, New York, NY, USA, 1992.

[8] K. Kobayashi, Y. Shestopalov, and Y. Smirnov, "Investigation of electromagnetic diffraction by a dielectric body in a waveguide using the method of volume singular integral equation," SIAM Journal on Applied Mathematics, vol. 70, no. 3, pp. 969-983, 2009.
[9] Y. V. Shestopalov and V. V. Lozhechko, "Direct and inverse problems of the wave diffraction by screens with arbitrary finite inhomogeneities," Journal of Inverse and Ill-Posed Problems, vol. 11, no. 6, pp. 643-654, 2003.

[10] E. Eves, K. Murphy, and V. Yakovlev, "Reconstruction of complex permittivity with neural network-controlled FDTD modeling," The Journal of Microwave Power and Electromagnetic Energy, vol. 41, p. 1317, 2007.

[11] Y. Shestopalov and Y. Smirnov, "Existence and uniqueness of a solution to the inverse problem of the complex permittivity reconstruction of a dielectric body in a waveguide," Inverse Problems, vol. 26, no. 10, Article ID 105002, 2010.

[12] Y. Shestopalov and Y. Smirnov, "Determination of permittivity of an inhomogeneous dielectric body in a waveguide," Inverse Problems, vol. 27, no. 9, Article ID 095010, 2011.

[13] L. Beilina and M. V. Klibanov, Approximate Global Convergence and Adaptivity for Coefficient Inverse Problems, Springer, New York, NY, USA, 2012.

[14] L. Beilina and M. V. Klibanov, "A new approximate mathematical model for global convergence for a coefficient inverse problem with backscattering data," Journal of Inverse and IllPosed Problems, vol. 20, no. 4, pp. 513-565, 2012.

[15] L. Beilina and M. V. Klibanov, "The philosophy of the approximate global convergence for multi-dimensional coefficient inverse problems," Complex Variables and Elliptic Equations, vol. 57, no. 2-4, pp. 277-299, 2012.

[16] L. Beilina, N. T. Thành, M. V. Klibanov, and M. A. Fiddy, "Reconstruction from blind experimental data for an inverse problem for a hyperbolic equation," Inverse Problems, vol. 30, no. 2, Article ID 025002, 2014.

[17] L. Beilina, N. T. Thành, M. V. Klibanov, and J. B. Malmberg, "Reconstruction of shapes and refractive indices from backscattering experimental data using the adaptivity," Inverse Problems, http://arxiv.org/abs/1404.5862.

[18] L. Beilina and M. V. Klibanov, "Reconstruction of dielectrics from experimental data via a hybrid globally convergent/adaptive inverse algorithm," Inverse Problems, vol. 26, no. 12, Article ID 125009, 2010.

[19] A. V. Kuzhuget, L. Beilina, M. V. Klibanov, A. Sullivan, L. Nguyen, and M. A. Fiddy, "Blind backscattering experimental data collected in the field and an approximately globally convergent inverse algorithm," Inverse Problems, vol. 28, no. 9, Article ID 095007, 2012.

[20] M. V. Klibanov, M. A. Fiddy, L. Beilina, N. Pantong, and J. Schenk, "Picosecond scale experimental verification of a globally convergent algorithm for a coefficient inverse problem," Inverse Problems, vol. 26, no. 4, Article ID 045003, 30 pages, 2010.

[21] N. T. Thành, L. Beilina, M. V. Klibanov, and M. A. Fiddy, "Reconstruction of the refractive index from experimental backscattering data using a globally convergent inverse method," SIAM Journal on Scientific Computing, vol. 36, no. 3, pp. B273-B293, 2014.

[22] N. T. Thành, L. Beilina, M. V. Klibanov, and M. A. Fiddy, "Imaging of buried objects from experimental backscattering radar measurements using a globally convergent inverse algorithm," http://arxiv.org/abs/1406.3500v1.

[23] M. Asadzadeh and L. Beilina, "A posteriori error analysis in a globally convergent numerical method for a hyperbolic 
coefficient inverse problem," Inverse Problems, vol. 26, no. 11, Article ID 115007, 115007 pages, 2010.

[24] L. Beilina and M. V. Klibanov, "Synthesis of global convergence and adaptivity for a hyperbolic coefficient inverse problem in 3D," Journal of Inverse and Ill-Posed Problems, vol. 18, no. 1, pp. 85-132, 2010.

[25] L. Beilina, "Adaptive finite element method for a coefficient inverse problem for Maxwell's system," Applicable Analysis, vol. 90, no. 10, pp. 1461-1479, 2011.

[26] L. Beilina and C. Johnson, "A posteriori error estimation in computational inverse scattering," Mathematical Models \& Methods in Applied Sciences, vol. 15, no. 1, pp. 23-35, 2005.

[27] L. Beilina and E. Karchevskii, "The layer-stripping algorithm for reconstruction of dielectrics in an optical fiber," in Inverse Problems and Applications, Springer Proceedings in Mathematics \& Statistics, 2014.

[28] E. M. Karchevskii, "Study of spectrum of guided waves of dielectric fibers," in Proceedings of the Mathematical Methods in Electromagnetic Theory (MMET '98), vol. 2, pp. 787-788, 1998.

[29] E. M. Karchevskii, "Analysis of the eigenmode spectra of dielectric waveguides," Computational Mathematics and Mathematical Physics, vol. 39, no. 9, pp. 1493-1498, 1999.

[30] E. M. Karchevskii, "The fundamental wave problem for cylindrical dielectric waveguides," Differential Equations, vol. 36, no. 7, pp. 1109-1111, 2000.

[31] A. O. Spiridonov and E. M. Karchevskiy, "Projection methods for computation of spectral characteristics of weakly guiding optical waveguides," in Proceedings of the International Conference Days on Diffraction (DD '13), pp. 131-135, May 2013.

[32] E. M. Karchevskii and S. I. Solov'ev, "Investigation of a spectral problem for the Helmholtz operator in the plane," Differential Equations, vol. 36, no. 4, pp. 631-634, 2000.

[33] E. M. Kartchevski, A. I. Nosich, and G. W. Hanson, "Mathematical analysis of the generalized natural modes of an inhomogeneous optical fiber," SIAM Journal on Applied Mathematics, vol. 65, no. 6, pp. 2033-2048, 2005.

[34] A. Frolov and E. Kartchevskiy, "Integral equation methods in optical waveguide theory," in Inverse Problems and Large-Scale Computations, vol. 52 of Springer Proceedings in Mathematics \& Statistics, pp. 119-133, 2013.

[35] E. Karchevskiy and Y. Shestopalov, "Mathematical and numerical analysis of dielectric waveguides by the integral equation method," in Proceedings of the Progress in Electromagnetics Research Symposium (PIERS '13), pp. 388-393, Stockholm, Sweden, August 2013.

[36] M. P. H. Andresen, H. E. Krogstad, and J. Skaar, "Inverse scattering of two-dimensional photonic structures by layer stripping," Journal of the Optical Society of America B: Optical Physics, vol. 28, no. 4, pp. 689-696, 2011.

[37] J. D. Joannopoulos, R. D. Meade, and J. N. Winn, Photonic Crystals: Molding the Flow of Light, Princeton University, Princeton, NJ, USA, 1995.

[38] L. A. Klein and C. T. Swift, "An improved model for the dielectr ic constant of sea water at mi- crowave frequencies," IEEE Transactions on Antennas and Propagation, vol. 25, no. 1, pp. 104-111, 1977.

[39] A. Stogryn, "Equations for calculating the dielectric constant of saline water," IEEE Transactions on Microwave Theory and Techniques, vol. 19, no. 8, pp. 733-736, 1971.
[40] M. Pastorino, Microwave Imaging, John Wiley \& Sons, Hoboken, NJ, USA, 2010.

[41] D. L. Colton and R. Kress, Integral Equation Methods in Scattering Theory, Pure and Applied Mathematics (New York), John Wiley \& Sons, New York, NY, USA, 1983.

[42] A. Neumaier, "Residual inverse iteration for the nonlinear eigenvalue problem," SIAM Journal on Numerical Analysis, vol. 22, no. 5, pp. 914-923, 1985. 

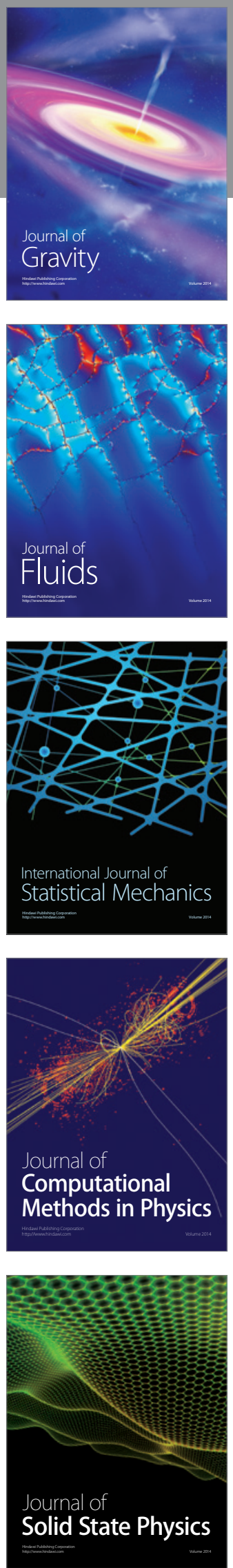

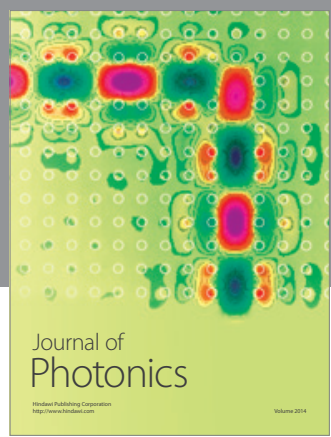

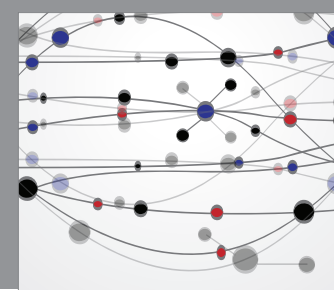

The Scientific World Journal

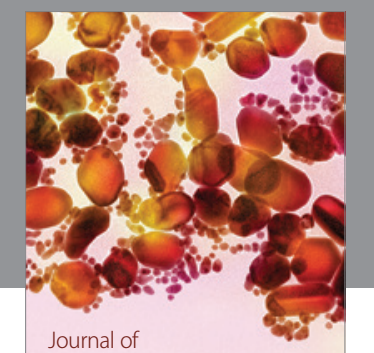

Soft Matter
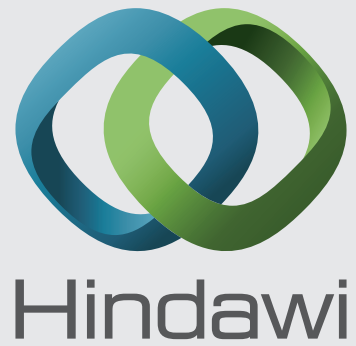

Submit your manuscripts at

http://www.hindawi.com
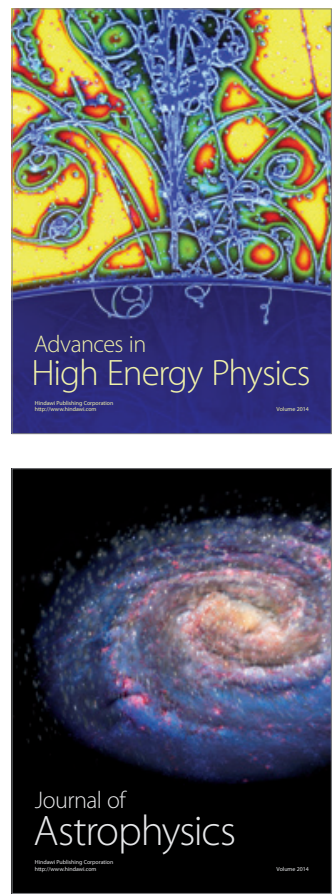
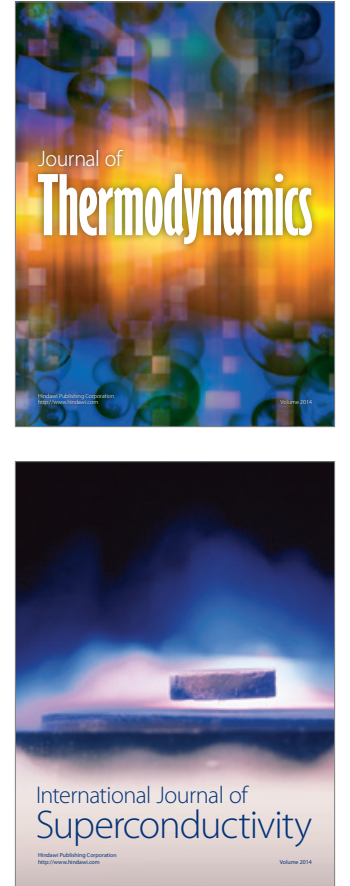
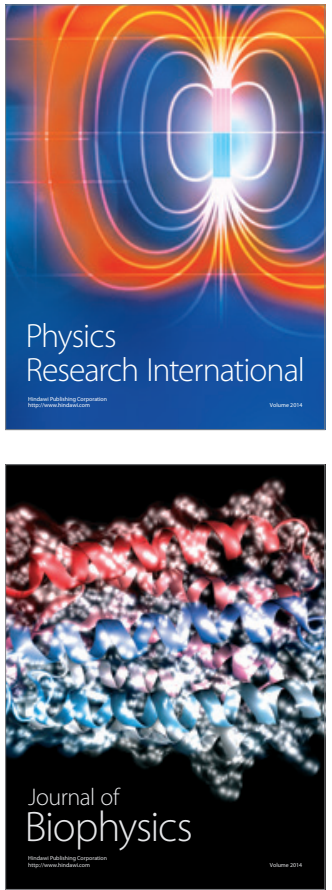
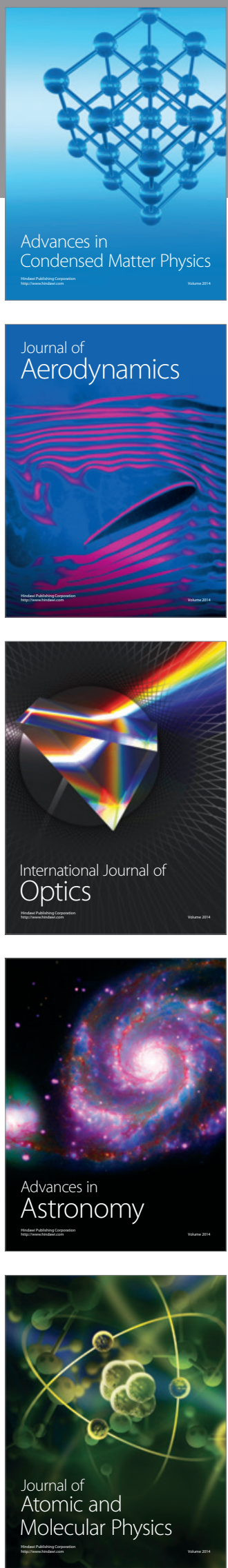